\title{
Möglichkeiten der
}

Ernährungstherapie bei
chronisch entzündlichen

Darmerkrankungen

Joachim Glaser

Nach derzeitigem Kenntnisstand haben Ernährungsgewohnheiten keinen signifikanten Einfluss auf die Pathogenese chronisch entzündlicher Darmerkrankungen (CED). Eine längere Stilldauer scheint Säuglinge jedoch vor späterer Erkrankung an CED zu schützen. Liegen im Zuge der Erkrankung Mangelzustände vor, ist eine spezielle Ernährungstherapie indiziert, vorzugsweise enteral - insbesondere bei Kindern.

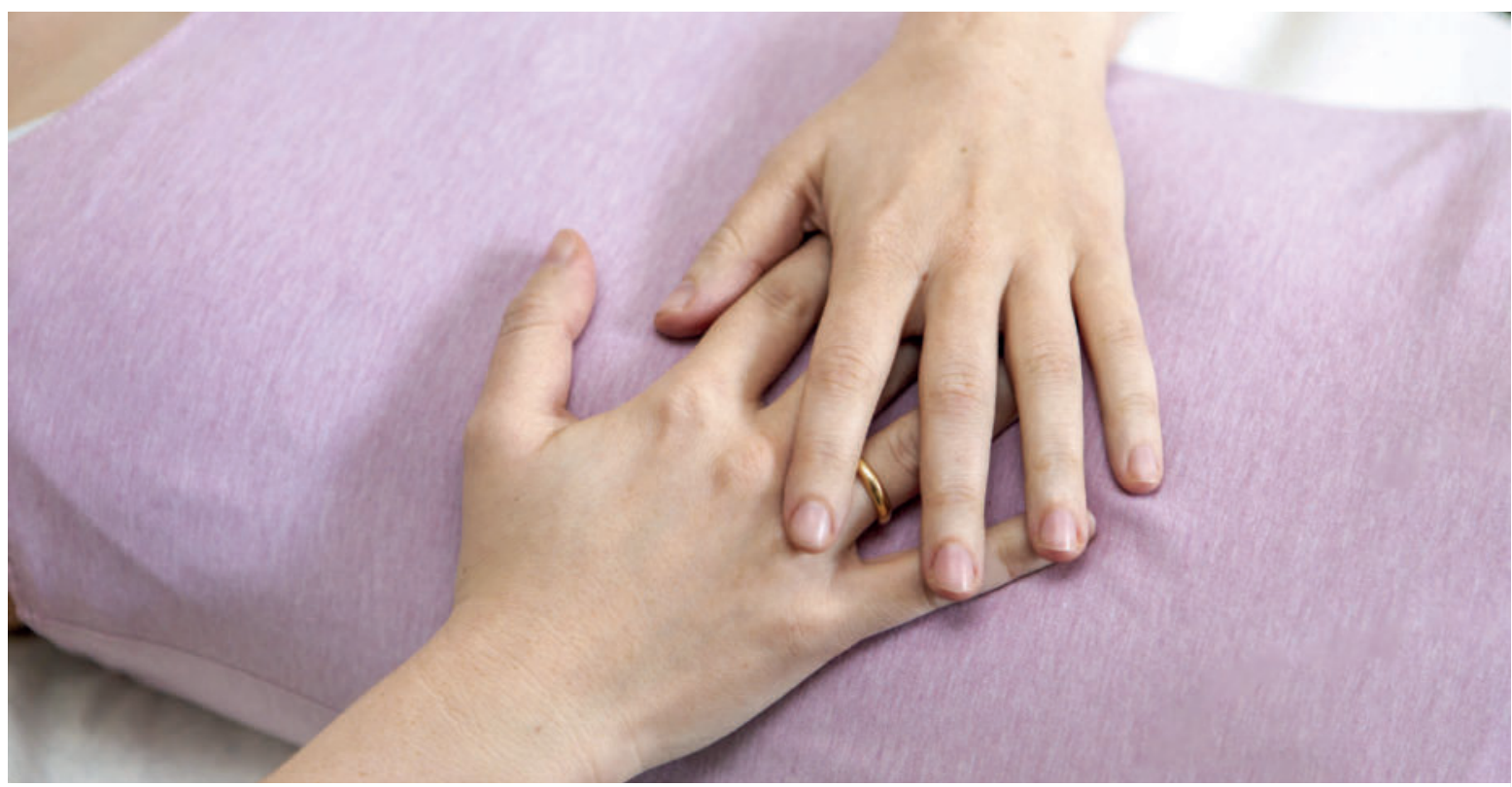

Bei Colitis ulcerosa und Morbus Crohn kann es mehrmals täglich zu Durchfällen mit krampfartigen Bauchschmerzen kommen, auch in der Nacht. Drohende Mangelernährung ist ein wichtiges Thema für die Ernährungsberatung. Quelle: Kirsten Oborny, Thieme Gruppe

Ernährung beginnt in der Regel mit der Nahrungsaufnahme über den Mund. Transport der Nahrung, Verdauung und Aufnahme der Nährstoffe finden im Gastrointestinaltrakt statt. Der Magen-Darm-Trakt, zentrales Erfolgsorgan der Ernährung, ist bei chronisch entzündlichen Darmerkrankungen (CED), Morbus Crohn und Colitis ulcerosa, direkt von entzündlichen Veränderungen betroffen. Die Frage, ob Ernährungsgewohnheiten bei der Genese von
CED eine Rolle spielen und in welchem Umfang sie gegebenenfalls Beschwerden und Krankheitsverlauf beeinflussen, ist daher prinzipiell berechtigt. Es ist auch zu erwarten, dass eine entzündlich bedingte, gestörte Resorptionskapazität des Darms und reduzierte Nahrungsaufnahme einen Nährstoffmangel zur Folge haben können. Ernährungsmedizinische Maßnahmen könnten bei CED zum Beispiel auf den Erhalt eines ausreichenden Ernährungszustands und 
die Besserung von Beschwerden abzielen. Darüber hinaus könnten möglicherweise auch die Krankheitsaktivität und der Krankheitsverlauf Ziele ernährungstherapeutischer Interventionen sein.

\section{Ernährung und Pathogenese von CED}

Wie chronisch entzündliche Darmerkrankungen exakt entstehen, ist bis heute nur unvollständig bekannt. Neben zentralen pathogenetischen Faktoren, wie beeinträchtigter Barriereschutzfunktion der Schleimhaut und einer pathologischen Immunreaktion, sind genetische Voraussetzungen und Umwelteinflüsse, speziell bei Morbus Crohn auch Tabakrauchinhalation, als Auslöser von Bedeutung.

Ob und inwieweit das Darmmikrobiom des Menschen einen Einfluss auf die Pathogenese von CED hat, wird derzeit vermehrt wissenschaftlich untersucht.

\section{Merke}

Speziell die Ernährung hat einen nicht unerheblichen Einfluss auf die Zusammensetzung der intestinalen Mikrobiota.

Ältere Studien ließen für die Pathogenese von CED eine Bedeutung des Umfangs der Aufnahme von raffiniertem Zucker, Industriefetten und tierischem Eiweiß sowie auch von vermindertem Verzehr an Ballaststoffen vermuten. Gesicherte Informationen über Ernährungsgewohnheiten, die eine CED auslösen oder gegebenenfalls auch vermeiden helfen könnten, liegen bis heute nicht vor. Eine längere Stilldauer (> 6 Monate) scheint Säuglinge jedoch tatsächlich vor späterer Manifestation einer CED zu schützen [1][2][3].

\section{Mangelernährung und Mikronährstoffmangel}

Häufiger werden bei akuten Schüben oder chronisch aktiven Krankheitsverläufen Symptome einer Mangelernährung beobachtet. Bei der Genese einer Malnutrition im Rahmen von CED spielen neben intestinalen Resorptionsstörungen und zu rascher Darmpassage mit Diarrhö auch Faktoren wie Inappetenz, Schmerzen und Übelkeit eine Rolle. Folge ist nicht selten eine reduzierte Nahrungsaufnahme bei gleichzeitig gesteigertem Energiebedarf. Bei Morbus Crohn (20-70\% der Fälle) kann Mangelernährung durchaus als typisches Symptom betrachtet werden, bei Colitis ulcerosa tritt sie weniger häufig auf [4]. Bei M. Crohn erhöht sich das Risiko einer Malnutrition durch ein postoperativ entstandenes Kurzdarmsyndrom gegebenenfalls zusätzlich [5].

Merke

Das Screening auf Mangelernährung ist bei der Therapie von CED sehr wichtig.
Ein Body-Mass-Index $(\mathrm{BMI})<18,5 \mathrm{~kg} / \mathrm{m}^{2}$, ein unbeabsichtigter Gewichtsverlust von > 10\% des Ausgangsgewichts in den letzten 3-6 Monaten oder ein $\mathrm{BMI}<20 \mathrm{~kg} / \mathrm{m}^{2}$ und unbeabsichtigter Gewichtsverlust $>5 \%$ in den letzten 3-6 Monaten gelten dabei als wichtige Hinweise auf eine Malnutrition. Der Verdacht sollte dann durch spezielle diagnostische Maßnahmen weiter abgeklärt werden, z. B. durch eine Bioimpedanzanalyse (BIA).

\section{PRAXISTIPP}

Hinweise auf Mangelernährung bei CED

- Body-Mass-Index (BMI) unter 18,5 kg/m²

- Gewichtsverlust von mehr als $10 \%$ in 3-6 Monaten

- BMl unter $20 \mathrm{~kg} / \mathrm{m}^{2}$ plus Gewichtsverlust von mehr als $5 \%$ in 3-6 Monaten

(modifiziert nach DGEM)

Es ist prognostisch wichtig, Symptome einer Mangelernährung möglichst früh zu erkennen, um schwerere Komplikationen zu vermeiden. In diesem Zusammenhang weist die aktuelle DGEM-Leitlinie auf die Notwendigkeit einer alljährlichen Diätberatung von CED-Patienten hin [6].

Bei Vorliegen einer Mangelernährung sind spezielle ernährungstherapeutische Interventionen indiziert. Diese beinhalten neben Anpassungen der Lebensmittelauswahl und Zwischenmahlzeiten den Einsatz hochkalorischer Trinknahrungen. Weil es über die Entzündung der Darmschleimhaut häufiger zu erheblichen Proteinverlusten kommt, kann eine zusätzliche Eiweißsubstitution erforderlich sein [7]. Wenn die orale Nahrungsaufnahme nicht ausreicht, sollte primär eine enterale Sondenernährung erfolgen. Gelingt eine ausreichende Ernährung mittels Trinkund Sondennahrung nicht, zum Beispiel bei Patienten mit Kurzdarmsyndrom, High-Output-Stoma, hohen Dünndarmfisteln oder vor geplanten operativen Maßnahmen, ist eine parenterale Ernährung notwendig [3][8][9][10].

\section{Spurenelemente und Vitamine}

Beschleunigte Darmpassage, gestörte Resorption sowie verminderte Nahrungsaufnahme haben bei CED häufiger auch einen Mangel an Mikronährstoffen zur Folge, der seinerseits Beschwerden verursachen kann ( $\triangleright$ Tab. 1).

Nicht selten wird eine Anämie infolge von Eisenmangel festgestellt. Neben chronischer Entzündung und Resorptionsstörungen können bei Colitis ulcerosa wiederkehrende Blutverluste zu Eisenmangel und Anämie beitragen. Häufig ist dann eine medikamentöse Eisensubstitution erforderlich, zumindest in der Remissionsphase kann eine orale Eisensubstitution ausreichen [3]. Gelingt eine ausreichende orale Substitution nicht, z. B. bei hoher entzündlicher Aktivität, sollte die Eisensubstitution parenteral erfolgen. 
\Tab. 1 Vorkommen von Mikronährstoffmangel bei CED (modifiziert nach [14]).

\begin{tabular}{|l|l|l|l|}
\hline Mikronährstoffmangel & Morbus Crohn & Colitis ulcerosa & mögliche Folgen \\
\hline Eisen & $25-53 \%$ & $55-81 \%$ & Anämie \\
\hline Folsäure & $10-62 \%$ & $36-60 \%$ & Anämie \\
\hline Vitamin B 12 & $3-48 \%$ & $5 \%$ & Anämie, Neuropathie \\
\hline Vitamin A & $11-50 \%$ & $16 \%$ & Nachtblindheit, trockene Haut \\
\hline Vitamin D & $25-75 \%$ & $35 \%$ & Osteoporose/Osteomalazie \\
\hline Kalzium & $13 \%$ & n. a. & Osteoporose, Krämpfe, Rhythmusstörungen \\
\hline Magnesium & $14-33 \%$ & $17-24 \%$ & Schwäche, Krämpfe, Rhythmusstörungen \\
\hline Zink & $1-40 \%$ & $10 \%$ & Haarausfall, Hautprobleme \\
\hline
\end{tabular}

Auch ein Folsäuremangel und insbesondere bei Morbus Crohn (z. B. nach lleozökalresektion) ein Vitamin-B 12 -Mangel können zur Anämie beitragen und eine Substitution erfordern.

Signifikante Verluste von elementarem Zink sind bei chronischer Diarrhö keine Seltenheit und können zu substitutionspflichtigem Zinkmangel führen. Weil Zink mit der intestinalen Eisen- und Kupferresorption interferiert [11], ist die Dauer der Substitution (meist oral) zeitlich zu begrenzen.

Eine gestörte Vitamin-D-Aufnahme, z. B. bei M. Crohn mit Dünndarmbefall, oder auch eine Glukokortikoidtherapie können einen Vitamin-D-Mangel mitverursachen. Es ist dann eine orale Vitamin-D-Substitution indiziert [3].

\section{PRAXISTIPP}

Eine möglichst zu vermeidende, längerdauernde Kortisontherapie sollte grundsätzlich mit einer Substitution von Vitamin D und ggf. auch Kalzium einhergehen.

Zuwenig wird meist beachtet, dass bei Patienten mit Mangelernährung jeder Ursache, z. B. auch auf dem Boden von CED, ein Vitamin $\mathbf{B}_{1}$-Mangel vorliegen kann. Es können dann die Zeichen einer chronischen oder sogar akuten Wernicke-Enzephalopathie auftreten wie Schwindel, Ataxie, demenzielle Symptome oder Verhaltensauffälligkeiten. Treten Symptome wie Schluckstörungen, Sehstörungen oder Sprachstörungen (Apraxie) hinzu, ist bei Mangelernährung durchaus von einer Wernicke-Enzephalopathie auszugehen [12][13] und eine hochdosierte Vitamin- $B_{1}$-Zufuhr indiziert (initial ggf. auch intravenös).

\section{Akute Entzündung}

In der akuten Entzündungsphase ( $>$ Abb. 1) wird in der Regel eine leichte, ballaststoffarme Ernährung empfohlen und in dieser Situation meist besser toleriert als eine

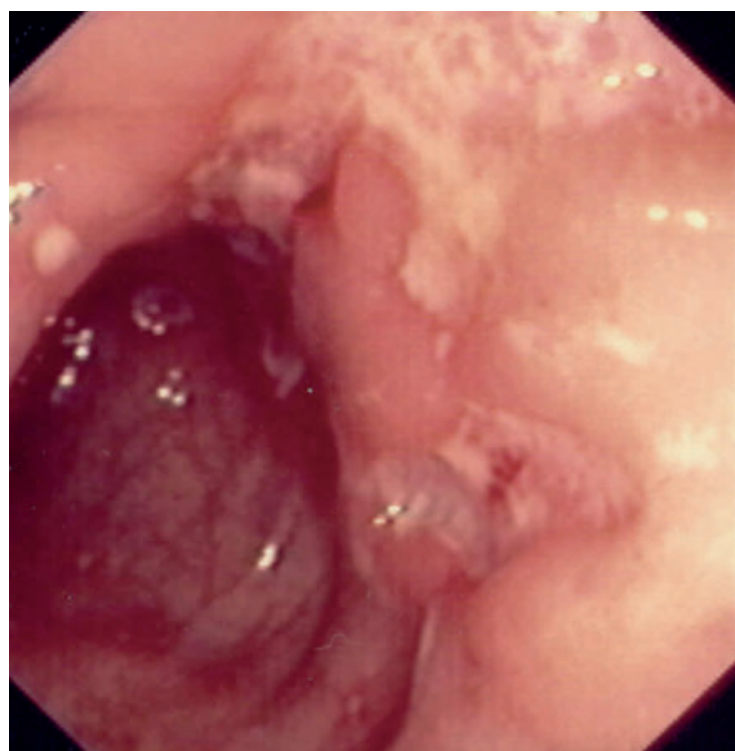

- Abb. 1 Tiefe längliche Geschwüre im Dickdarm bei Morbus Crohn. Quelle: Autor

normale Mischkost. Allerdings ist ein relevanter Einfluss von ballaststoffarmer Ernährung auf die Krankheitsaktivität bislang nicht durch Evidenz gesichert.

Eine Ernährungstherapie kann bei akuter Entzündung z. B. auf die Besserung eines reduzierten Ernährungszustandes abzielen. Dies schafft gegebenenfalls bessere Voraussetzungen für den weiteren Krankheitsverlauf und die medikamentöse Behandlung [14], insbesondere bei Patienten mit Morbus Crohn.

\section{Morbus Crohn}

Eine Ernährungstherapie mit nährstoffdefinierten oder chemisch definierten Nährlösungen (Trinknahrung und Sondenkost) wirkt sich bei Morbus Crohn häufig auch günstig auf die Krankheitsaktivität aus [15]. Bei Kindern und Jugendlichen mit M. Crohn und akuter Entzündung ist sie einer Steroidtherapie gleichwertig und wird in dieser Altersgruppe als primäre therapeutische Maßnahme eingesetzt [16][17]. 


\section{Merke}

Bei Erwachsenen ist eine Kortisonbehandlung im akuten Entzündungsschub wirksamer als die Ernährungstherapie [18][19].

Deshalb wird eine enterale Ernährung mit Trinknahrung oder Sondenkost bei Erwachsenen mit M. Crohn und hoher Krankheitsaktivität nicht als Primärtherapie empfohlen, kann aber ergänzend zur medikamentösen Therapie eingesetzt werden [5].

\section{Colitis ulcerosa}

In der akuten Entzündungsphase der Colitis ulcerosa ist eine signifikante Wirkung der Ernährungstherapie auf die Krankheitsaktivität nicht gesichert. Da jedoch im akuten Schub der Nährstoffbedarf generell erhöht ist, kann eine Ernährungstherapie auch bei Colitis ulcerosa zur Sicherung einer ausreichenden Nährstoffversorgung in Erwägung gezogen werden, wenn Risiken für eine Mangelernährung vorliegen [3]. Die enterale Ernährungstherapie ist dabei einer parenteralen Ernährung vorzuziehen.

\section{Parenterale Ernährung}

Indikationen für eine parenterale Ernährung ergeben sich bei CED eher in speziellen Situationen, wie z. B. Kurzdarmsyndrom, komplexen Fisteln und Darmobstruktion sowie perioperativ [5], vor allem wenn eine genügende enterale Nährstoffversorgung nicht gelingt [6].

\section{Remission}

In der Remission ist der Ernährungsstatus bei chronisch entzündlichen Darmerkrankungen meist normal. Eine Ernährungstherapie zur Verbesserung der Ernährungssituation ist in der Remission daher in der Regel nicht notwendig. Verlässliche Belege für die Annahme, dass besondere ernährungsmedizinische Maßnahmen bei CED zum Remissionserhalt beitragen könnten, gibt es bislang nicht. Dies gilt bei normalem Ernährungsstatus auch für den M. Crohn in der Remission [5].

Merke

Bei Hinweisen auf eine Mangelernährung ist auch in der Remission eine Ernährungstherapie in Erwägung zu ziehen.

Individuelle Nahrungsmittelunverträglichkeiten sind bei CED in der Remission grundsätzlich zu berücksichtigen. Liegt parallel eine Laktoseintoleranz vor, ist eine laktosearme Ernährung indiziert. Stichhaltige Belege dafür, dass Eliminationsdiäten einen relevanten Einfluss auf die Dauer der Remission haben, fehlen bislang [5][20][21].

Eine Evidenz für einen remissionsverlängernden Effekt von präbiotischen, ballaststoffreichen Lebensmitteln liegt ebenfalls nicht vor [5][22]. Einzelne kontrollierte Studien weisen darauf hin, dass sich eine sogenannte
Low-FODMAP-Diät (LFD) in der CED-Remission günstig auf postinflammatorische Reizdarmbeschwerden auswirken kann [23].

Probiotika, speziell Bakterienpräparate mit E. coli Nissle, sind in der Remission leichterer Verläufe einer Colitis ulcerosa ähnlich wirksam wie Mesalazin [24]. Für Kombinationsprobiotika wie VSL\#3 wurden positive Effekte insbesondere bei Patienten mit rezidivierender Pouchitis berichtet [3], eine abschließende Bewertung erscheint bislang noch nicht möglich.

\section{Merke}

Zur remissionserhaltenden Therapie des M. Crohn sind Probiotika nicht geeignet.

Zusammenfassend kommen spezielle ernährungsmedizinische Maßnahmen in der CED-Therapie vor allem bei Mangelernährung und im akuten Entzündungsschub zur Anwendung, in der Remissionsphase einer CED sind sie meist entbehrlich.

\section{KERNAUSSAGEN}

- Eine längere Stilldauer scheint Säuglinge vor späterer Erkrankung an CED zu schützen.

- Bei Vorliegen einer Mangelernährung ist eine Ernährungstherapie indiziert. Gelingt eine ausreichende orale Zufuhr nicht, ist eine enterale Sondenernährung erforderlich, ggf. auch eine parenterale Ernährung.

- Mikronährstoffmängel erfordern eine Substitution.

- Im akuten Entzündungsschub wird eine leicht verdauliche, ballaststoffarme Ernährung meist besser toleriert als eine normale Mischkost. Die Ernährungstherapie mit Trinknahrung oder Sondenkost gilt als Behandlungsoption in der akuten Entzündungsphase des M. Crohn, wird jedoch nur bei Kindern und Jugendlichen im akuten Entzündungsschub als primäre Behandlungsmaßnahme empfohlen. Sie kann bei Erwachsenen mit Morbus Crohn und florider Entzündung aber eine notwendige medikamentöse Therapie ergänzen.

- Für die Colitis ulcerosa ist ein Effekt der Ernährungstherapie nicht gesichert, die Indikation bleibt hier Krankheitsphasen mit deutlich erhöhtem Nährstoffbedarf und dem Risiko einer Mangelernährung vorbehalten.

- Solange eine genügende Versorgung mit Nährstoffen gewährleistet ist, sollte die enterale Ernährungstherapie einer parenteralen Ernährung vorgezogen werden.

- Besondere ernährungsmedizinische Maßnahmen sind bei normalem Ernährungszustand in der Remission meist nicht erforderlich. 


\section{Interessenkonflikt}

Der Autor gibt an, dass kein Interessenkonflikt besteht.

\section{Über den Autor}

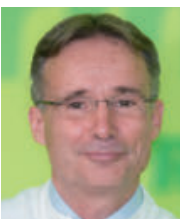

\section{Prof. Dr. med. Joachim Glaser}

Prof. Dr. med. Joachim Glaser ist Chefarzt der Vitalisklinik Bad Hersfeld, Fachklinik und Rehabilitationszentrum für Verdauungs- und Stoffwechselkrankheiten.

\section{Korrespondenzadresse}

Prof. Dr. med. Joachim Glaser

Vitalisklinik Bad Hersfeld GmbH

Am Weinberg 3

36251 Bad Hersfeld

E-Mail: joachim.glaser@vitalisklinik.de

\section{Literatur}

[1] Klement E, Reif S. Breastfeeding and risk of inflammatory bowel disease. Am J Clin Nutr 2005; 82: 486

[2] Gearry RB, Richardson AK, Frampton CM et al. Population based cases control study of inflammatory bowel disease risk factors. J Gastroenterol Hepatol 2010; 25: 325-333

[3] Kucharzik T, Dignass AU, Atreya R et al. Aktualisierte S3-Leitlinie Colitis ulcerosa der Deutschen Gesellschaft für Gastroenterologie, Verdauungs-und Stoffwechselkrankheiten (DGVS). Z Gastroenterol 2018; 56: 1087-1169

[4] Siegmann-Thoss C, van Dillen V, Keller R. Ernährung und Entzündung-Empfehlungen zur Ernährung bei chronischentzündlichen Darmerkrankungen (CED). Endo-Praxis 2019; 35: 83-89

[5] Fuchssteiner H, Nigl K, Mayer A, Kristensen B et al. Ernährung und chronisch entzündliche Darmerkrankungenein Konsensus der Arbeitsgruppe chronisch entzündliche Darmerkrankungen der Österreichischen Gesellschaft für Gastroenterologie und Hepatologie (Austrian Guidelines for nutrition in IBD). Z Gastroenterol 2014; 52: 376-386

[6] Bischoff SC, Koletzko B, Lochs H et al. S3-Leitlinie der Deutschen Gesellschaft für Ernährungsmedizin (DGEM), Klinische Ernährung in der Gastroenterologie (Teil 4) - Chronisch-entzündliche Darmerkrankungen. Aktuel Ernahrungsmed 2014; 39: e72-e98

[7] Massironi S, Rossi RE, Cavalcoli FA et al. Nutritional deficiences in inflammatory bowel disease: therapeutic approaches. Clin Nutr 2013; 32: 904-910

[8] Rombeau JL, Barot LR, Williamson CE et al. Preoperative parenteral nutrition and surgical outcome in patients with inflammatory bowel disease. Am J Surg 1982; 143: 139-143

[9] Sorensen J, Kondrup J, Prokopowicz J et al. EuroOOPS: an international, multicenter study to implement nutritional risk screening and evaluate clinical outcome. Clin Nutr 2008; 27: $340-349$

[10] Weimann A, Breitenstein S, Breuer JP et al. Clinical nutrition in surgery. Guidelines of the German Society for Nutritional Medicine. Chirurg 2014; 85: 320-326

[11] Rossi RE, Whyand T, Murray CD et al. The role of dietary supplements in inflammatory bowel disease: a systematic review. Europ J Gastroenterol Hepatol 2016; 28: 1357-1364

[12] Glöckler TM, Wunderlich O, Jabs B et al. Die Wernicke-Enzephalopathie. Ärzteblatt Sachsen 2016; Heft 9: 382-385

[13] Bozbara A, Coskun H, Ozarmagan S et al. A rare complication of adjustable gastric banding: Wernicke's encephalopathy. Obesity surgery 2000; 10: 274-275

[14] Meier R. Ernährung bei chronisch entzündlichen Darmerkrankungen (CED). Aktuel Ernahrungsmed 2012; 37: 349-362

[15] Leiß O. Ernährungsfaktoren in Genese und Therapie chronisch-entzündlicher Darmerkrankungen (Morbus Crohn und Colitis ulcerosa). Verdauungskrankheiten 2004; 22: 319-337

[16] Heuschkel RB, Menache CC, Megerian T] et al. Enteral nutrition and corticosteroids in the treatment of acute Crohn's disease in children. J Pediatr Gastroenterol Nutr 2000; 31: 8-15

[17] Dziechziarz P, HorvathA, Shamir R et al. Meta-analysis: enteral nutriton in active Crohn's disease in children. Aliment Pharmacol Ther 2007; 26: 795-803

[18] Trallori SG, D`Albasio G, Milla M et al. Defined-formula diets versus steroids in the treatment of active Crohn's disease: a meta-analysis. Scand J Gastroenterol 1996; 31: 267-272

[19] Zachos M, Tondeur M, Griffiths AM. Enteral nutritional therapy for induction of remission in Crohn's disease. Cochrane Database Syst Rev 2017; 24: CD000542

[20] Brandes JW, Lorenz-Meyer H. Sugar free diet: a new perspective in the treatment of Crohn disease? Randomized, control study. Z Gastroenterol 1981; 19: 1-12

[21] Ritchie JK, Wadsworth J, Lennard-Jones JE et al. Controlled multicenter therapeutic trial of an unrefined carbohydrate, fibre rich diet in Crohn's disease. Br Med J (Clin res Ed) 1987; 295: 517-520

[22] Wedlake L, Slack N, Andreyev JN et al. Fiber in the treatment and maintenance of inflammatory bowel disease: A systematic review of randomized controlled trials. Inflamm Bowel Dis 2014; 20: $576-586$

[23] Zhan YL, Zhan YA, Dai SX. Is a low FODMAP diet beneficial for patients with inflammatory bowel disease? A meta-analysis and systematic review. Clin Nutr 2018; 37: 123-129

[24] Kruis W, Fric P, Pokrotnieks ] et al. Maintaining remission of ulcerative colitis with the probiotic Escherichia coli Nissle 1917 is as effective as with standard mesalazine. Gut 2004; 53: $1617-1623$

\section{Bibliografie}

DOI https://doi.org/10.1055/a-1115-9515

Ernährung \& Medizin 2020; 35: 107-111

(c) 2020. Thieme. All rights reserved.

Georg Thieme Verlag KG, Rüdigerstraße 14,

70469 Stuttgart, Germany

ISSN 1439-1635 